\title{
Patterns of the Exclusive Double Diffraction
}

\author{
Petrov V.A. and Ryutin R.A. \\ Institute for High Energy Physics \\ 142 281 Protvino, Russia
}

\begin{abstract}
We consider Exclusive Double Diffractive Events (EDDE) as a powerfull tool to study the picture of the $p p$ interaction. Calculations of the cross-sections for the process $p+p \rightarrow p+M+p$ are presented in the convenient form for further experimental applications. We propose measurements of t-distributions in the joint CMS-TOTEM experiment. It is shown that important information on the interaction region could be extracted from the diffractive pattern.
\end{abstract}

\section{Keywords}

Exclusive Double Diffractive Events - Pomeron - Regge-Eikonal model - Higgs Radion - Jets - Diffractive Pattern 


\section{Itroduction}

With the first LHC run coming closer the hopes for confirmation of various theory predictions get heated. The huge amount of works is related to the search of fundamental particles of the Standardt Model or its extensions (Higgs boson, Superpartners, gravitons and so on) and to the investigations of so called "hard" QCD processes, which correspond to very short space-time scales. "Soft" diffractive proceses take in this raw its own, distinctive place.

LHC collaborations aimed at working in low and high $p_{T}$ regimes related to typical undulatory (diffractive) and corpuscular (point-like) behaviours of the corresponding crosssections may offer a very exciting possibility to observe an interplay of both regimes [1]. In theory the "hard part" can be (hopefully) treated with perturbative methods whilst the "soft" one is definitely nonperturbative.

Below we give several examples of such an interplay: exclusive particle production by diffractively scattered protons, i.e. the processes $p+p \rightarrow p+M+p$, where + means also a rapidity gap and $\mathrm{M}$ represents a particle or a system of particles consisting of or strongly coupled to the two-gluon state [2].

These processes are related to the dominant amplitude of exclusive and semiinclusive two-gluon production. Driving mechanism of the diffractive processes is the Pomeron. Data on the total cross-sections demands unambiguosly for the Pomeron with largerthan-one intercept, thereof the need to take into account the "soft" rescattering (i.e. "unitarisation").

EDDE gives us unique experimental possibilities for particle searches and investigations of diffraction itself. This is due to several advantages of the process: a) clear signature of the process; b) possibility to use "missing mass method" that improve the mass resolution; c) background is strongly suppressed; d) spin-parity analysis of the central system can be done; e) interesting measurements concerning the interplay between "soft" and "hard" scales are possible. All these properties can be realized in common CMS/TOTEM detector measurements at LHC [3].

\section{Exclusive double diffraction}

The exclusive double diffractive process is related to the dominant amplitude of the exclusive two-gluon production. Driving mechanism of this processes is the Pomeron.

To calculate an amplitude of the EDDE, we use an approach which was considered in detail in Ref. [2]. In the framework of this approach, the amplitude can be sketched as shown in Fig. 1. After the tensor contraction of the amplitudes $T_{1,2}$ with the gluon-gluon fusion vertex, the full "bare" amplitude $T_{M}$ depicted in Fig. 1 looks like

$$
T_{M}=\frac{2}{\pi} c_{g p}^{2} e^{b\left(t_{1}+t_{2}\right)}\left(-\frac{s}{M^{2}}\right)^{\alpha_{P}(0)} F_{g g \rightarrow M} I_{s}
$$




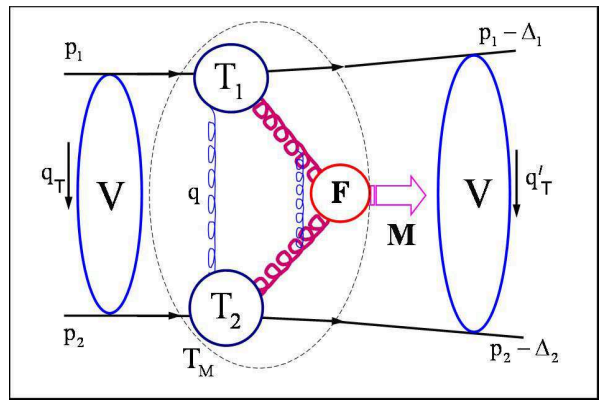

Figure 1: Model for EDDE

Here

$$
\begin{aligned}
b & =\alpha_{P}^{\prime}(0) \ln \left(\frac{\sqrt{s}}{M}\right)+b_{0}, \\
b_{0} & =\frac{1}{4}\left(\frac{r_{p p}^{2}}{2}+r_{g p}^{2}\right),
\end{aligned}
$$

with the parameters of the "hard" Pomeron trajectory, that appears to be the most relevant in our case, presented in Table 1. The last factor in the r.h.s. of (1) is

$$
I_{s}=\int_{0}^{\mu^{2}} \frac{d l^{2}}{l^{4}} F_{s}\left(l^{2}, \mu^{2}\right)\left(\frac{l^{2}}{s_{0}+l^{2} / 2}\right)^{2 \alpha_{P}(0)},
$$

where $l^{2}=-q^{2} \simeq \mathbf{q}^{2}, \mu=M / 2$, and $s_{0}$ is a scale parameter of the model which is also used in the global fitting of the data on $p p(p \bar{p})$ scattering for on-shell amplitudes [1]. The fit gives $s_{0} \simeq 1 \mathrm{GeV}^{2}$. If we take into account the emission of virtual "soft" gluons, while prohibiting the real ones, that could fill rapidity gaps, it results in a Sudakov-like suppression [4]:

$$
\begin{aligned}
F_{s}\left(l^{2}, \mu^{2}\right) & =\exp \left[-\int_{l^{2}}^{\mu^{2}} \frac{d p_{T}^{2}}{p_{T}^{2}} \frac{\alpha_{s}\left(p_{T}{ }^{2}\right)}{2 \pi} \int_{\Delta}^{1-\Delta} z P_{g g}(z) d z+\int_{0}^{1} \sum_{q} P_{q g}(z) d z\right], \\
P_{g g}(z) & =6 \frac{(1-z(1-z))^{2}}{z(1-z)}, \\
\Delta & =\frac{p_{T}}{p_{T}+\mu} .
\end{aligned}
$$

The off-shell gluon-proton amplitudes $T_{1,2}$ are obtained in the extended unitary approach [5]. The "hard" part of the EDDE amplitude, $F_{g g \rightarrow M}$, is the usual gluon-gluon fusion amplitude calculated perturbatively in the SM or in its extensions.

The data on total cross-sections demand unambiguously the Pomeron with larger-thanone intercept, thereof the need in unitarization. The amplitude with unitary corrections, 


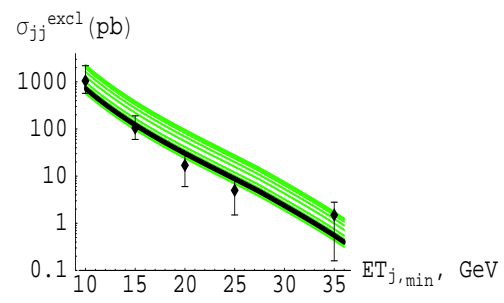

Figure 2: The latest data from CDF and predictions for EDDE.

Table 1: Phenomenological parameters of the "hard" Pomeron trajectory obtained from the fitting of the HERA and Tevatron data (see [2], [6]), and data on $p p(p \bar{p})$ scattering [1]. The value of the $c_{g p}$ is corrected in accordance with the latest data from CDF [7], which is depicted in the Fig. 2 with the range of possible curves.

\begin{tabular}{||c|c|c|c|c||}
\hline$\alpha_{P}(0)$ & $\alpha_{P}^{\prime}(0), \mathrm{GeV}^{-2}$ & $r_{p p}^{2}, \mathrm{GeV}^{-2}$ & $r_{g p}^{2}, \mathrm{GeV}^{-2}$ & $c_{g p}$ \\
\hline 1.203 & 0.094 & 2.477 & 2.54 & $3.2 \pm 0.5$ \\
\hline
\end{tabular}

$T_{M}^{u n i t}$, are depicted in Fig. 1. It is given by the following analytical expressions:

$$
\begin{aligned}
T_{M}^{\text {unitar }}\left(p_{1}, p_{2}, \Delta_{1}, \Delta_{2}\right) & =\frac{1}{16 s s^{\prime}} \int \frac{d^{2} \mathbf{q}_{T}}{(2 \pi)^{2}} \frac{d^{2} \mathbf{q}_{T}^{\prime}}{(2 \pi)^{2}} V\left(s, \mathbf{q}_{T}\right) \\
& \times T_{M}\left(p_{1}-q_{T}, p_{2}+q_{T}, \Delta_{1 T}, \Delta_{2 T}\right) V\left(s^{\prime}, \mathbf{q}_{T}^{\prime}\right), \\
V\left(s, \mathbf{q}_{T}\right) & =4 s(2 \pi)^{2} \delta^{2}\left(\mathbf{q}_{T}\right)+4 s \int d^{2} \mathbf{b} e^{i \mathbf{q}_{T} \mathbf{b}}\left[e^{i \delta_{p p \rightarrow p p}}-1\right],
\end{aligned}
$$

where $\Delta_{1 T}=\Delta_{1}-q_{T}-q_{T}^{\prime}, \Delta_{2 T}=\Delta_{2}+q_{T}+q_{T}^{\prime}$, and the eikonal function $\delta_{p p \rightarrow p p}$ can be found in Ref. [1]. Left and right parts of the diagram in Fig. 1b denoted by $V$ represent "soft" rescattering effects in initial and final states, i.e. multi-Pomeron exchanges. As was shown in [8], these "outer" unitary corrections strongly reduce the value of the corresponding cross-section and change an azimuthal angle dependence.

In the equation (11) we present only the Born terms from amplitudes $T_{1,2}$. It is sufficient for $\left|t_{1,2}\right|<1 \mathrm{GeV}$ due to fast decrease of the differential cross-section in $t_{1,2}$, and the contribution of these corrections to the total cross-section are less than several percents. But when we consider the diffractive pattern in the region of $1<\left|t_{1,2}\right|<5 \mathrm{GeV}$, we have to take into account rescattering corrections inside the amplitudes $T_{1,2}$. In this case $I_{s}$ in 


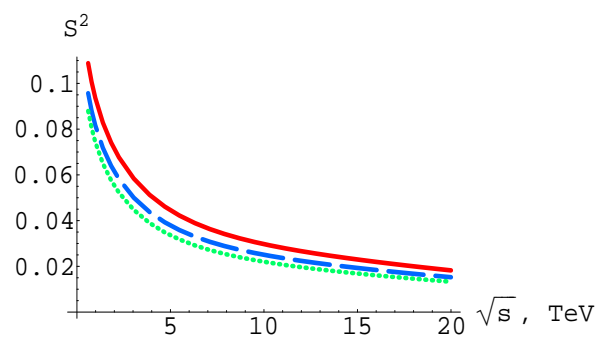

Figure 3: "Soft" survival probability $S^{2}$ as a function of $\sqrt{s}$ for masses of the central system $10 \mathrm{GeV}$ (solid curve), $50 \mathrm{GeV}$ (dashed one) and $200 \mathrm{GeV}$ (dotted one).

the equation (1) changes to the following expression:

$$
\begin{aligned}
I_{s}^{c o r r} & =\int_{0}^{\mu^{2}} \frac{d l^{2}}{l^{4}} F_{s}\left(l^{2}\right)\left(\frac{l^{2}}{s_{0}+l^{2} / 2}\right)^{\alpha_{P}\left(t_{1}\right)+\alpha_{P}\left(t_{2}\right)}\left(1+h\left(v, t_{1}\right)\right)\left(1+h\left(v, t_{2}\right)\right), \\
h(v, t) & =\sum_{n=2}^{\infty} \frac{(-1)^{n-1}}{n ! \cdot n}\left(\frac{c_{g p}}{8 \pi b_{1}(v)} \exp \left[-\frac{i \pi\left(\alpha_{P}(0)-1\right)}{2}\right] v^{\alpha_{P}(0)-1}\right)^{n-1} . \\
& \cdot \exp \left[\frac{b_{1}(n-1)}{n}|t|\right], \\
v & =\frac{\sqrt{s}}{M} \frac{l^{2}}{s_{0}+l^{2} / 2},
\end{aligned}
$$

and $b$ to

$$
b_{1}=\alpha_{P}^{\prime}(0) \ln v+b_{0} .
$$

To calcuate differential and total cross-sections for exclusive processes we can use the formula

$$
\begin{aligned}
\left.M^{2} \frac{d \sigma^{E D D E}}{d M^{2} d y d \Phi_{g g \rightarrow M}}\right|_{y=0} & =\hat{L}^{E D D E} \frac{d \hat{\sigma}^{J_{z}=0}}{d \Phi_{g g \rightarrow M}}, \\
\hat{L}^{E D D E} & =\frac{c_{g p}^{4}}{2^{5} \pi^{6}}\left(\frac{s}{M^{2}}\right)^{2\left(\alpha_{P}(0)-1\right)} \frac{1}{4 b^{2}} I_{s} S^{2}, \\
S^{2} & =\frac{\int d^{2} \vec{\Delta}_{1} d^{2} \vec{\Delta}_{2}\left|T_{M}^{\text {unitar }}\right|^{2}}{\int d^{2} \vec{\Delta}_{1} d^{2} \vec{\Delta}_{2}\left|T_{M}\right|^{2}}
\end{aligned}
$$

where $d \hat{\sigma}^{J_{z}=0} / d \Phi_{g g \rightarrow M}$ is the "hard" exclusive singlet gluon-gluon fusion cross-section and $S^{2}$ is the so called "soft" survival probability. In this work the quantity $\hat{L}$ is called $g^{I P} g^{I P}$ luminocity.

The factor $S^{2}$ is depicted in the Fig. 3 for the systems M with quantum numbers $0^{++}$ (SM Higgs boson, Radion, jet-jet). For other cases it is of the same order and can be calculated using the Monte-Carlo event generator EDDE [9]. 


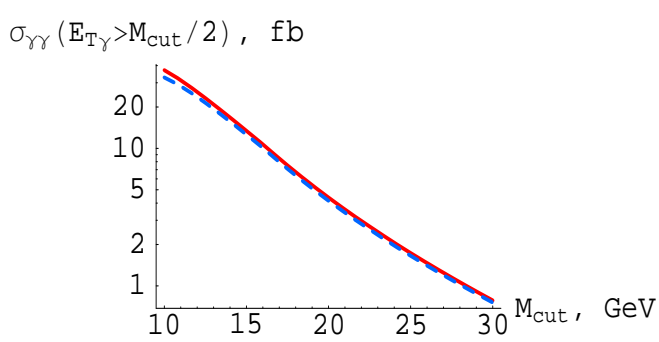

(a)

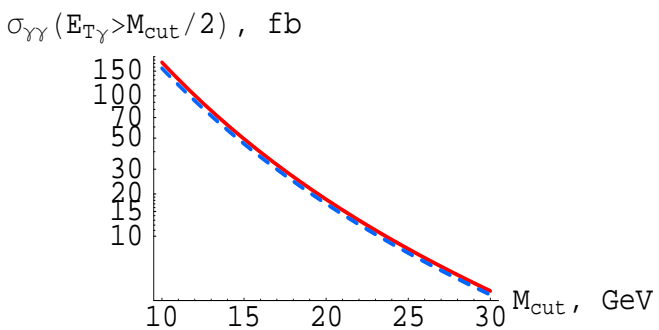

(c)

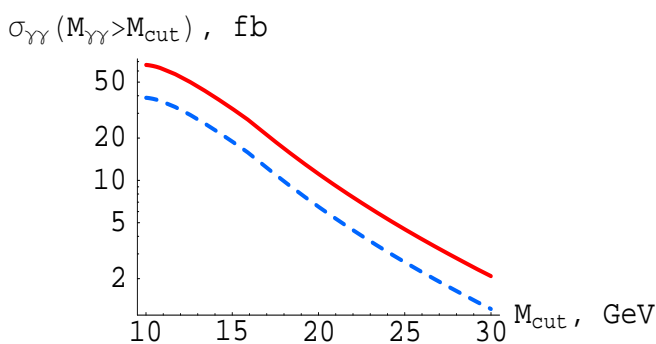

(b)

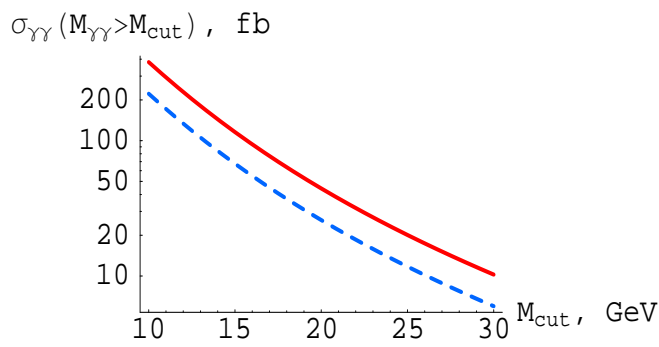

(d)

Figure 4: Cross-sections for the process $p p \rightarrow p+\gamma \gamma+p$ for different kinematical cuts. Solid and dashed curves correspond to the pseudorapidity cuts $\left|\eta_{\gamma}\right|<2$ and $\left|\eta_{\gamma}\right|<1$. a) $\sqrt{s}=1.8 \mathrm{TeV}$, CDF cuts for $\xi_{1,2}$ [7], and cut on the $E_{T \gamma}$; b) $\sqrt{s}=1.8 \mathrm{TeV}, \mathrm{CDF}$ cuts for $\xi_{1,2}$ [7], and cut on the $M_{\gamma \gamma}$; c) $\sqrt{s}=14 \mathrm{TeV}$, symmetric cuts $0.0003<\xi_{1,2}<0.1$, and cut on the $E_{T \gamma}$; d) $\sqrt{s}=14 \mathrm{TeV}$, symmetric cuts $0.0003<\xi_{1,2}<0.1$, and cut on the $M_{\gamma \gamma}$.

\section{Results}

First of all we would like to discuss some features of the process $p p \rightarrow p+\gamma \gamma+p$, since this process is the standard one to obtain the model parameters. Cross-sections for this process are presented in the Fig. 4 . It is important to note that the cut $E_{T \gamma}>E_{\text {cut }}=M_{\text {cut }} / 2$ is used in the major part of experimental works, that is why we have to use the same one in our calculations. But in some theoretical works [10] $E_{T}>E_{\text {cut }}$ means another cut $M_{\gamma \gamma}>2 E_{c u t}$, which leads to the result, similar to the one presented in the Fig. $4 \mathrm{~b}$. In this figure cross-section for $\left|\eta_{\gamma}\right|<2$ is about two times higher than for $\left|\eta_{\gamma}\right|<1$. Such difference is only possible in the kinematics, when $M_{\gamma \gamma}>2 E_{\text {cut }}$. It follows from rather simple calculations. Total cross-section for the process $g g \rightarrow \gamma \gamma$ can be represented as [11]

$$
\hat{\sigma}_{g g \rightarrow \gamma \gamma}^{J_{z}=0}\left(M_{\gamma \gamma}, \eta_{\max }\right)=C_{\gamma \gamma} F\left(\eta_{\max }\right) \frac{\alpha_{s}\left(M_{\gamma \gamma} / 2\right)^{2}}{M_{\gamma \gamma}^{2}},
$$

where $\eta_{\max }$ is the pseudorapidity cut in the central mass frame of the diphoton system, $C_{\gamma \gamma}$ is the constant,

$$
F\left(\eta_{\max }\right)=\int_{-\eta_{\max }}^{\eta_{\max }} \frac{d \eta}{\operatorname{ch}^{2} \eta}\left[1+\left(1-2 \eta \operatorname{th} \eta+\frac{1}{4}\left(\pi^{2}+4 \eta^{2}\right)\left(1+\operatorname{th}^{2} \eta\right)\right)^{2}\right]
$$



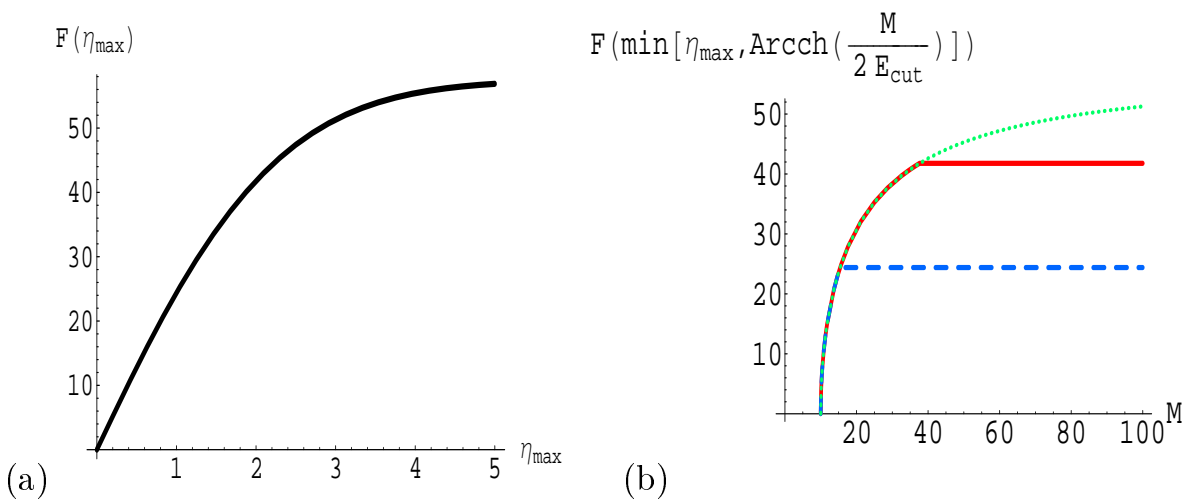

(b)

Figure 5: a) Function $F\left(\eta_{\max }\right)$; b) $\eta_{\max }=2$ (solid curve), $\eta_{\max }=1$ (dashed one), $F\left(\operatorname{Arcch} \frac{M}{2 E_{\text {cut }}}\right)$ (dotted one), $E_{\text {cut }}=5 \mathrm{GeV}$.

is depicted in the Fig. 5a. And for the process $p p \rightarrow p+\gamma \gamma+p$ from (14) we have

$$
\sigma_{p p \rightarrow p+\gamma \gamma+p}\left(E_{c u t}, \eta_{\max }\right) \simeq \int_{2 E_{c u t}}^{\sqrt{\xi_{1 \max } \xi_{2 \max }}} \frac{d M^{2}}{M^{2}} \hat{L}^{E D D E}(M) \hat{\sigma}_{g g \rightarrow \gamma \gamma}^{J_{z}=0}\left(M, \eta_{\max }\right) \Delta y .
$$

We are interested in the ratio of total cross-sections for different $\eta_{\max }$. Let us consider first the kinematics with cuts

$$
M_{\gamma \gamma}>2 E_{c u t},\left|\eta_{\gamma}\right|<\eta_{\max }
$$

In this case

$$
\frac{\sigma_{p p \rightarrow p+\gamma \gamma+p}\left(M>2 E_{c u t},|\eta|<2\right)}{\sigma_{p p \rightarrow p+\gamma \gamma+p}\left(M>2 E_{c u t},|\eta|<1\right)} \simeq \frac{F(2)}{F(1)} \simeq 1.7
$$

Since in the central mass frame of the diphoton system we have $M_{\gamma \gamma}=2 E_{T \gamma}$ ch $\eta_{\gamma}$, in the kinematics with

$$
E_{T \gamma}=\frac{M_{\gamma \gamma}}{2 \operatorname{ch} \eta_{\gamma}}>E_{c u t},\left|\eta_{\gamma}\right|<\eta_{\max }
$$

we have additional cut

$$
\left|\eta_{\gamma}\right|<\operatorname{Arcch} \frac{M_{\gamma \gamma}}{2 E_{c u t}}
$$

and we should use $F\left(\min \left[\eta_{\max }, \operatorname{Arcch} \frac{M_{\gamma \gamma}}{2 E_{c u t}}\right]\right)$ instead of $F\left(\eta_{\max }\right)$ in (17). This new function is shown in the Fig. 5b. The main contribution to the integral comes from small masses due to fast decrease in $M$, but in this region we have the same cut $|\eta|<\operatorname{Arcch} \frac{M}{2 E_{\text {cut }}}$ for different $\eta_{\max }$. And it is easy to get the following result

$$
\frac{\sigma_{p p \rightarrow p+\gamma \gamma+p}\left(E_{T}>E_{c u t},|\eta|<2\right)}{\sigma_{p p \rightarrow p+\gamma \gamma+p}\left(E_{T}>E_{c u t},|\eta|<1\right)} \simeq 1.1 \text { for } E_{c u t}=5 \mathrm{GeV} .
$$

Even if we take $\alpha_{s}=$ const and $\hat{L}^{E D D E}=$ const, we will get the ratio 1.3 , and not $\sim 2$ as in [10]. This simple example shows that we should be carefull with the kinematics during 
our calculations, since this could lead to different predictions. Now we can compare our result with the latest data on the exclusive $\gamma \gamma$ production from CDF [12]

$$
\sigma_{p p \rightarrow p+\gamma \gamma+p}\left(E_{T \gamma}>5 \mathrm{GeV},\left|\eta_{\gamma}\right|<2\right)=0.14_{-0.04}^{+0.14} \text { (stat.) } \pm 0.03 \text { (syst.) pb . }
$$

This prediction is higher than our calculations based on the di-jet production [7]. If we beleive in this result, the model parameter $c_{g p}$ is at least $20 \%$ higher, than our estimations. The possible reason is that in $\gamma \gamma$ production we use the region of small masses for the normalization of our parameter (and higher masses for the di-jet production), but the uncertainty in the scale dependence of the cross-section is rather large (factor $\sim 2$ ). This result from CDF may serve a good signal for the future exclusive Higgs boson production, since it makes the cross-section higher by about two times.

Now we can estimate the backgrounds for the exclusive Higgs production at LHC [2]. Rates for these processes at the integrated luminocity $100 \mathrm{fb}^{-1}$ are summarized in the table 2 (parameter $c_{g p}=3.7$, i.e. we use more optimistic values based on the data on the di-jet production [7]). From this table we can obtain signal to background ratio $\sim 1$. More exact estimations will be made in the nearest future after full Monte-carlo simulations.

Table 2: Rates for the exclusive Higgs production and different backgrounds at the integrated luminocity $100 \mathrm{fb}^{-1}$ and $\Delta M_{\text {missing }}=4 \mathrm{GeV}$. The probability to misidentify gluon jets with b-jets is taken to be $1 \%$.

\begin{tabular}{|c|c|}
\hline process & N events \\
\hline$\sigma^{E D D E}(H \rightarrow b b)$ & 27 \\
\hline$\sigma^{S I}(H \rightarrow b b)$ & 2 \\
\hline$\sigma^{E D D E}(b b)$ & 12 \\
\hline$\sigma^{E D D E}(b b g)$ & 1 \\
\hline$\sigma^{E D D E}(g g) \cdot 10^{-4}$ & 14 \\
\hline$\sigma^{E D D E}(g g g) \cdot 10^{-4}$ & 2 \\
\hline
\end{tabular}

\section{Diffractive patterns}

In the general agenda of the LHC experiment diffraction often looks as an "auxiliary tool" for other processes such as Higgs boson and exotics searches, background supression and so on. Nevertheless, diffractive measurements have their own classical tasks directly related to the angular (or $t$ ) distributions.

Diffractive pattern is usually characterized by the peak at small values of $t$, and complicated structure with dips or breaks and bumps for larger $t$ [1]. This picture reflects the ondulatory properties of quantum processes as contrasted to more habitual particle-like behaviour, and allows us to get an information about the size and shape of the strong interaction region at large distances (i.e. directly related to confinement of the QCD colour fields). 
- From the diffractive pattern we extract model independent parameters of the interaction region such as the $t$-slope which is $R^{2} / 2$, with $R$ the transverse radius of the interaction region.

- We can also estimate the longitudinal size of the interaction region [13]:

$$
\Delta x_{L}>\frac{\sqrt{s}}{2 \sqrt{<t^{2}>-<t>^{2}}}
$$

The longitudinal interaction range is somehow "hidden" in the amplitude but it is this range that is responsible for the "absorption strength". A rough analogue is the known expression for the radiation absorption in media which critically depends on the thickness of the absorber.

- The very presence of dips is the signal of the quantum interference of hadronic waves.

- The depth of dips is determined by the real part of the scattering amplitude

According to the data from $S p \bar{p} S$ and Tevatron the transverse radius of the interaction region is of order of $\left.1.2 \mathrm{fm} \simeq 1.5<r_{\mathrm{em}}\right\rangle$. The longitudinal size can be estimated from the second inequality (26) and is of order of $2800 \mathrm{fm}$.

Diffractive pattern moves due to changes in kinematical parameters like the energy of the interaction or an additional hard scale. This motion reflects the dynamics of the process. The increase of the $t$-slope with energy reflects the growth of the interaction radius. At fixed collision energy the diffractive pattern is fixed as well.

However if we have in our disposal an additional hard scale we can operate the diffractive pattern adjusting this hard scale at our will and making, e.g., the interaction region larger or smaller.

Hard scale is related to small distances and, from the simple optical point of view, the pattern should move towards large values of $-t$ with the increase of the hard scale. HERA provides an excellent opportunity to observe the influence of a hard scale $\left(Q^{2}\right)$ on the diffractive pattern: the slope decreases with $Q^{2}$ in exclusive vector meson or photon production or for mesons containing heavy quarks $(J / \Psi)$ as contrasted to those composed of the light quarks (see Fig. 6) [14.

The decrease of the slope with $Q^{2}$ in electroproduction was predicted qualitatively in Ref. [15]: J.D. Bjorken argued that the decrease of the slope would be bounded from below by the size of nucleon [16]. The latter feature seems to be violated in the HERA data [17]. We have to mention that the presence of a high-mass particle in the final state does not always lead to the phenomena described above. For example, hadronic resonances with large masses have large size due to intrinsic motion of constituents, and can not be considered as a hard probe. In this case we have inverse dynamics of the pattern [18. This certainly is not the case for the processes considered below as they are related exclusively to short-distance probes, i.e. "high mass" means always "high $\mathrm{E}_{\mathrm{T}}$ ".

The diffractive pattern for the process $p+p \rightarrow p+j j+p$ as predicted on the basis of ref. [2] is displayed on the Fig. 7 where $\frac{1}{\sigma} \frac{d \sigma}{d t}$ means the exclusive differential cross section with all final variables integrated except one of the proton transverse momenta $(-t)$ and 


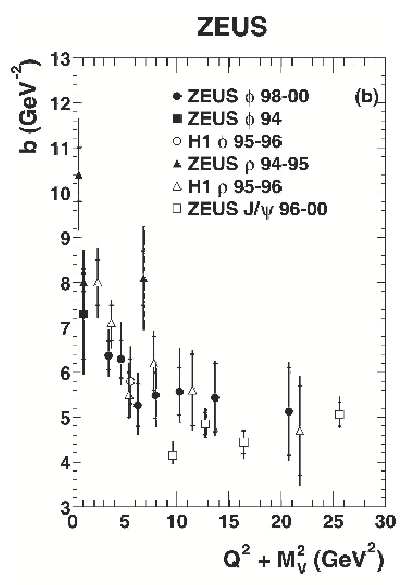

Figure 6: The slope b, as a function of $Q^{2}+M_{V}^{2}$, compared to other ZEUS and H1 results.

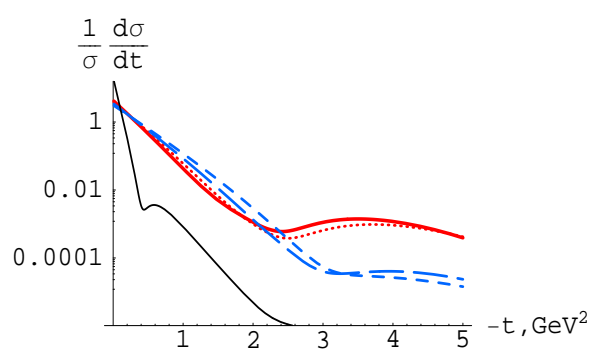

Figure 7: Normalised cross section for exclusive dijet production as a function of $t$ for $M_{X}=30 \mathrm{GeV}$ (the solid and long-dashed curves correspond to the LHC and TEVATRON energies, respectively) and $M_{X}=200 \mathrm{GeV}$ (the dotted and short-dashed curves correspond to the LHC and TEVATRON energies, respectively). The left curve corresponds to the elastic scattering at the LHC.

the value of the central mass $\left(\mathrm{M} \simeq 2 \mathrm{E}_{\mathrm{T}}\right)$. With two exclusive high- $\mathrm{E}_{\mathrm{T}}$ jets the expected dips will reflect the elastic scattering of the protons off the hard gluon. Their positions are shifted to the right in comparison with the proton-proton elastic scattering, as depicted on the Fig. 7. Such a shift is a clear signal of the short-distance scale due to jets.

Measurements of t-distributions and their dynamics in the exclusive central diffraction could be used for the proposed investigations. To obtain the detailed diffractive pattern with dips for $1 \mathrm{GeV}^{2}<-t<5 \mathrm{GeV}^{2}$ we need at least $10^{4}$ events for fixed (or falling within the small enough range of values) masses of the central system and $t$-resolution less than $10 \%$ in this region. At high luminosities the use of the missing mass method is limited below by central masses above $30 \mathrm{GeV}$ because of the acceptance limitations and the absence of resonances with high rates in this region. That is why the only way is to use exclusive or Semi-Inclusive (exclusive+"soft" radiation in the central rapidity region) dijet production. The best case is the measurements at the nominal luminosity at $\beta^{*}=0.5$. Results are summarized in the table 3 . 
Table 3: Rates for exclusive and semi-inclusive $\left(\left|\eta_{\text {soft }}\right|<5\right)$ double diffractive dijet production for luminosity $10^{33} \mathrm{~cm}^{-2} \mathrm{~s}^{-1}$ for different intervals of the invariant mass of the central system, $M_{X}$.

\begin{tabular}{|c|c|c|c|}
\hline $\mathrm{M}_{1}<\mathrm{M}_{X}<\mathrm{M}_{2}(\mathrm{GeV})$ & $t$-slope $\left(\mathrm{GeV}^{-2}\right)$ & $\mathrm{N}_{\text {ex }}$ & $\mathrm{N}_{\text {semi-incl. }}$ \\
\hline $29<\mathrm{M}_{X}<31$ & 4.6 & $2 \cdot 10^{4} /$ day & $6 \cdot 10^{4} /$ day \\
\hline $98<\mathrm{M}_{X}<102$ & 4.3 & $9 \cdot 10^{3} /$ month & $4.5 \cdot 10^{4} /$ month \\
\hline $196<\mathrm{M}_{X}<204$ & 4.1 & $5.5 \cdot 10^{3} /$ year & $4 \cdot 10^{4} /$ year \\
\hline
\end{tabular}

\section{Aknowledgements}

This work is supported by grants RFBR-06-02-16031 and INTAS-05-112-5481.

\section{References}

[1] V. A. Petrov,A. V. Prokudin, Eur.Phys.J. C23 (2002) 135.

[2] V.A. Petrov and R.A. Ryutin, JHEP 0408 (2004) 013.

[3] K. Eggert, M. Oriunno and M. Bozzo, TOTEM Technical Design Report, CERNLHCC-2004-002;

M. Deile, Talk given at the Workshop "Physics at LHC" (July 2004, Vienna), arXive:hep-ex/0503042.

[4] V.A. Khoze, A.D. Martin and M.G. Ryskin, Eur. Phys. J. C 14 (2000) 525; ibid. C 21 (2001) 99.

[5] V.A. Petrov, Talk given at the 6th Workshop on Elastic and Diffractive Scattering (20-24 Jun 1995, Blois, France). In Blois 1995, Frontiers in strong interactions, p. $139-143$.

[6] V.A. Petrov, A.V. Prokudin and R.A. Ryutin, Czech. J. Phys. 55 (2005) 17.

[7] CDF Coll.: C. Mesropian, FERMILAB-CONF-06-464-E;

K. Goulianos, FERMILAB-CONF-06-464-E.

[8] V.A. Petrov, R.A. Ryutin, A.E. Sobol and J.-P. Guillaud, JHEP 0506 (2005) 007.

[9] V.A. Petrov, R.A. Ryutin, A.E. Sobol and J.-P. Guillaud, arXiv:0711.1794 [hep-ph].

[10] V.A. Khoze, A.D. Martin, M.G. Ryskin, W.J. Stirling, Eur. Phys. J. C 38 (2005) 475.

[11] R. Gastmans, T.T. Wu, "The Ubiquitous photon: Helicity method for QED and QCD". Oxford, UK: Clarendon (1990) 648 p. 
[12] M. Albrow, "Central Exclusive Higgs Production", talk presented at CMS week, June, 2006.

[13] V. A. Petrov,A. V. Prokudin,S. M. Troshin and N. E. Tyurin, J.Phys. G 27 (2001) 2225 .

[14] ZEUS Collab., arXiv:hep-ex/0504010

[15] H. Cheng and T. T. Wu, Phys. Rev. 183 (1969) 1324.

[16] J. D. Bjorken. SLAC Report No SLAC-PUB-0905 (1971).

[17] R. Roland arXiv:hep-ex/0605096.

[18] G. Goggi et al. Phys.Lett. B79 (1978) 165. 